\title{
Colestasis en el adulto: enfoque diagnóstico y terapéutico. Revisión de tema
}

\section{Diagnostic and therapeutic approach for cholestasis in the adult}

Andrés José Gómez Aldana, MD, ${ }^{*}$ Mónica Tapias, MD, ${ }^{2}$ Adán Lúquez Mindiola, MD. ${ }^{3}$

\begin{abstract}
Médico Internista, Universidad Javeriana. Gastroenterólogo, Universidad Nacional de Colombia. Especialista en servicio de endoscopia y trasplante hepático, Fundación Santa Fe de Bogotá. Docente de la Facultad de Medicina, Universidad de los Andes. Bogotá, Colombia.

2 Médica internista. Hepatóloga, Fundación Santa Fe de Bogotá, Colombia.

3 Médico internista. Gastroenterólogo, Universidad Nacional de Colombia; Centro de Enfermedades Digestivas, GutMédica. Bogotá, Colombia.

*Correspondencia: Andrés José Gómez Aldana, andresgomezmd@hotmail.com
\end{abstract}

Fecha recibido: $16 / 03 / 19$

Fecha aceptado: 18/12/19

\begin{abstract}
Resumen
La colestasis es uno de los motivos de consulta más frecuentes en hepatología. Se genera por una alteración en la síntesis, la secreción o el flujo de la bilis, a través del tracto biliar. Esta se define por una elevación de enzimas como la fosfatasa alcalina (Alkaline Phosphatase, ALP) y la gamma-glutamil transferasa, y en estadios tardíos con la hiperbilirrubinemia, al igual que con otras manifestaciones clínicas, tales como el prurito y la ictericia.

El enfoque diagnóstico implica establecer el origen de dicha elevación, determinando si es intrahepática o extrahepática. Si es intrahepática, se debe esclarecer si proviene de los hepatocitos o de la vía biliar de pequeño y de gran calibre. El tratamiento dependerá de la etiología, por lo cual es importante un diagnóstico preciso. En esta revisión se presenta la fisiopatología y un enfoque diagnóstico y terapéutico.
\end{abstract}

\section{Palabras clave \\ Colestasis, intrahepática, extrahepática.}

\begin{abstract}
Cholestasis is one of the most frequent reasons for hepatology consultation. It is generated by altered synthesis, secretion or flow of bile through the biliary tract and is defined by elevated levels of enzymes such as alkaline phosphatase and gamma glutamyl transferase. In late stages, hyperbilirubinemia and clinical manifestations such as pruritus and jaundice develop. The diagnostic approach involves establishment of the reasons for elevated enzyme levels and determination of whether it is intrahepatic or extrahepatic. If it is intrahepatic, the source must be determined (hepatocytes, small bile ducts, or large caliber bile ducts). Treatment depends on the etiology, so accurate diagnosis is important. This review presents the pathophysiology and a diagnostic and therapeutic approach.
\end{abstract}

\section{Keywords}

Cholestasis, intrahepatic, extrahepatic.

\section{INTRODUCCIÓN}

El término colestasis proviene de las palabras griegas chole, que significa bilis, y stasis, que traduce quieto. Esta definición involucra el síndrome generado por la alteración en la síntesis, la secreción o el flujo de la bilis, a través del tracto biliar. Se manifiesta a partir de la elevación de forma inicial de los niveles de la fosfatasa alcalina (Alkaline phosphatase, ALP) sérica y la gamma-glutamil transferasa (1), así como con la presencia de prurito generalizado sin lesiones en la piel y fatiga. Aunque la ictericia es un signo importante en la colestasis, puede estar ausente, particularmente en adultos con enfermedades crónicas colestásicas que pueden ser asintomáticos (2). 
Esta entidad se ha clasificado en el segmento involucrado en la excreción de la bilis, desde la etiología intrahepática, en la cual se evidencia un compromiso en el citoplasma hepatocelular y en los conductos biliares de tamaño mediano (hasta $400 \mu \mathrm{m}$ de diámetro), y las causas extrahepáticas, que comprometen los conductos biliares de gran tamaño. En consecuencia, las causas que generan la obstrucción de la vía biliar son producidas por cálculos, tumores pancreáticos o biliares, así como por metástasis hiliares (3).

\section{FISIOPATOLOGÍA}

Para comprender el origen de la colestasis, se debe partir desde la composición del lobulillo hepático. Esta unidad surge de la porción funcional más pequeña en el hígado, donde los hepatocitos se organizan en placas a lo largo del flujo sanguíneo, desde la vena porta hasta la vena central. Dentro de estas placas, los hepatocitos forman una luz tubular llamada el canalículo, en la cual se da la formación inicial de la bilis.

Estos hepatocitos contienen dos sistemas de toma y exportación, localizados en la porción basolateral (sinusoidal) y canalicular (apical) de la membrana de los hepatocitos y los colangiocitos, acompañados por un proceso osmótico que permite la generación de un flujo de agua que produce la secreción de bilis, desde los conductos biliares más pequeños hasta los más grandes (4-6) (Figura 1).

Los ácidos biliares captados ingresan por medio de receptores del péptido cotransportador de sodio-taurocolato

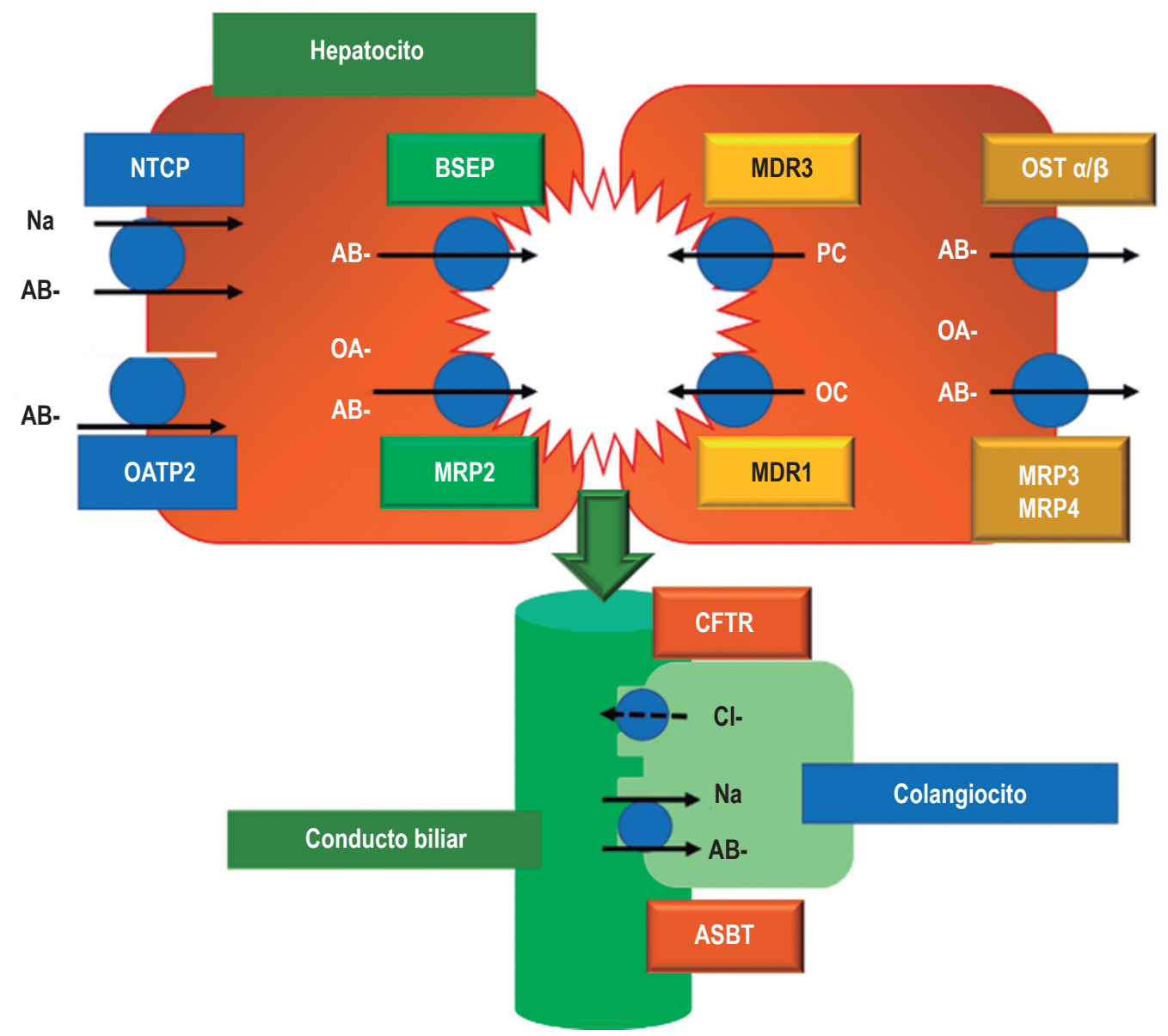

Figura 1. Sistemas de transporte de ácidos biliares. NTCP: péptido cotransportador de sodio-taurocolato (Na-Taurocholate Cotransporting Polypeptide); OATP2: polipéptido transportador de aniones orgánicos 2 (Organic Anion Transporting Polypeptide); BSEP: bomba exportadora de sales biliares (Bile Salt Export Pump); MRP2: proteína de resistencia a múltiples fármacos tipo 2 (Multidrug Resistance-associated Protein 2); MDR3: resistencia a múltiples fármacos tipo 3 (Multidrug Resistance 3); MDR1: resistencia a múltiples fármacos tipo 1 (Multidrug Resistance 1); OST a/b: transportador orgánico de solutos a/b (Organic Solute Transporter alpha and beta); MRP3: proteína de resistencia a múltiples fármacos tipo 3 (Multidrug Resistance-associated Protein 2); MRP4: proteína de resistencia a múltiples fármacos tipo 4 (Multidrug Resistance-associated Protein 4); CFTR: regulador transmembrana de la fibrosis quística (Cystic Fibrosis Transmembrane conductance Regulator); ASBT: transportador apical de sales biliares dependiente de sodio (Apical Sodium-Bile acid Transporter); AB: ácido biliar; OA: anión orgánico (Organic Anion); PC: fosfatidilcolina (Phosphatidylcholine). Tomada de la referencia 5. 
(Na-Taurocholate Cotransporting Polypeptide, NTCP), los cuales permiten concentrar las sales biliares en el hepatocito (6-8), así como otros polipéptidos transportadores de aniones orgánicos (Organic Anion Transporting Polypeptide, OATP2/OATP1B1), localizados en las membranas basolaterales de los hepatocitos (5).

Los ácidos biliares son excretados en la bilis por la bomba exportadora de ácidos biliares (Bile Salt Export Pump, BSEP) canaliculares y por la proteína exportadora canalicular conjugada MRP2 (Multidrug Resistance-associated Protein 2).

El transportador MRP2 (ABCC2) secreta aniones orgánicos como la bilirrubina conjugada, el glutatión e incluso antibióticos como la ceftriaxona (6). Otros transportadores localizados en la membrana canalicular son el MDR1 (Multidrug Resistance 1), la glicoproteína P (ABCB1) -que bombea componentes orgánicos y medicamentos catiónicos o con carga neutra fuera de la célula- y el MDR3 (Multidrug Resistance 3), el cual excreta fosfolípidos como la fosfatidilcolina que, en combinación con el colesterol y los ácidos biliares, forma las micelas (4-6) (Figura 1).

La bilis que se ha formado con las sales biliares primarias (colato y quenodesoxicólico), por la modificación enzimática del colesterol, confiere características más hidrofílicas y conforma las micelas, cuya función primordial es la toma de lípidos del intestino $(8,9)$. Sin embargo, son potencialmente peligrosas para la integridad de la membrana celular en condiciones de colestasis (6).

Entre tanto, los colangiocitos permiten la formación de la vía biliar y proveen otra serie de receptores que facilitan la reabsorción de ácidos biliares como el transportador apical de ácidos biliares dependiente de sodio (Apical SodiumBile acid Transporter, ASBT) y el canal de cloro CFTR (Cystic Fibrosis Transmembrane conductance Regulator), que es el regulador transmembrana de fibrosis quística (5).

Una vez las sales biliares se han secretado en el lumen intestinal, se recapturan por circulación enterohepática en el íleon, mediante un ASBT (9), hasta llegar al hepatocito, donde son nuevamente tomadas por el receptor NTCP, el cual permite concentrar las sales biliares en el hepatocito (5, 6). Asimismo, en la membrana basolateral del hepatocito se localizan otros receptores denominados MRP3 (Multidrug Resistance-associated Protein 3) y MRP4 (Multidrug Resistance-associated Protein 4), así como el transportador orgánico de solutos (Organic Solute Transporter alpha and beta, OST a/b). Estos son una ruta de excreción alternativa para los ácidos biliares y otros aniones orgánicos y se dirigen hacia la circulación sistémica (5).

La colestasis resulta de un defecto funcional en la formación de la bilis en el hepatocito, o de una alteración en la secreción y el flujo de esta a nivel de los conductos biliares $(4,5)$ (Figura 2).

\section{MANIFESTACIONES CLÍNICAS}

La colestasis puede tener un espectro de síntomas generados por la acumulación de sustancias en el hígado y en la sangre, que generalmente son excretados en la bilis. Los pacientes con este síndrome pueden encontrarse asintomáticos e incluso referir prurito e ictericia. Sin embargo, la ictericia puede estar ausente en adultos con enfermedades hepáticas colestásicas crónicas (2).

Un hallazgo importante es la esteatorrea, que se define como la pérdida $>10 \mathrm{~g}$ de grasa fecal por día, luego de una ingesta de $70 \mathrm{~g} / \mathrm{d}$ (10). Este fenómeno es secundario a una concentración inadecuada de bilis posprandial en el intestino delgado, lo que genera una malabsorción de grasas y vitaminas liposolubles que usualmente ayudan en la absorción de estos elementos $(3,9)$. La esteatorrea puede acompañarse de pérdida de peso, así como de la presencia de acropaquía o dedos hipocráticos (2).

Este déficit de vitaminas liposolubles tiene un amplio rango de síntomas neurológicos como ceguera nocturna (deficiencia de vitamina A); hiporreflexia o ataxia, como síntomas secundarios a una mielopatía (déficit de vitamina E); coagulopatía (deficiencia de vitamina K), y la presencia de trastornos del sistema osteomuscular con osteomalacia, osteoporosis y fracturas (deficiencia de vitamina D y calcio) $(3,9,10)$. Algunos estudios han sugerido que la hiperbilirrubinemia persistente $>2-3 \mathrm{mg} / \mathrm{dL}$ se asocia con la deficiencia de vitaminas liposolubles (11-13).

Además, se ha documentado la asociación de la osteoporosis y la deficiencia de vitaminas liposolubles con la prolongación del tiempo de protrombina, dado que los factores 2, 7,9 y 10 son dependientes de la vitamina $K$, la cual no puede ser absorbida durante los episodios de colestasis (14).

Asimismo, algunas enfermedades asociadas con la colestasis, como la colangitis biliar primaria, pueden continuar su progresión hasta presentar manifestaciones de hipertensión portal, dadas por ascitis, encefalopatía y hemorragia de las vías digestivas altas (10). Así, durante el examen físico es posible hallar xantomas (depósitos de colesterol en los tendones o prominencias óseas en los codos y las rodillas) o xantelasmas (depósitos de lípidos en los pliegues periorbitarios) $(2,11)$.

\section{ALTERACIONES PARACLÍNICAS}

El examen más sensible para la identificación de la colestasis es la medición de ácidos biliares séricos. Sin embargo, este estudio no está disponible con frecuencia. Por tanto, es necesario acudir a los marcadores bioquímicos de colestasis crónica, que son la ALP y la gamma-glutamil transferasa (2).

La fosfatasa puede elevarse por causas fisiológicas asociadas a un ascenso de dicha enzima. Esta elevación ocurre en 


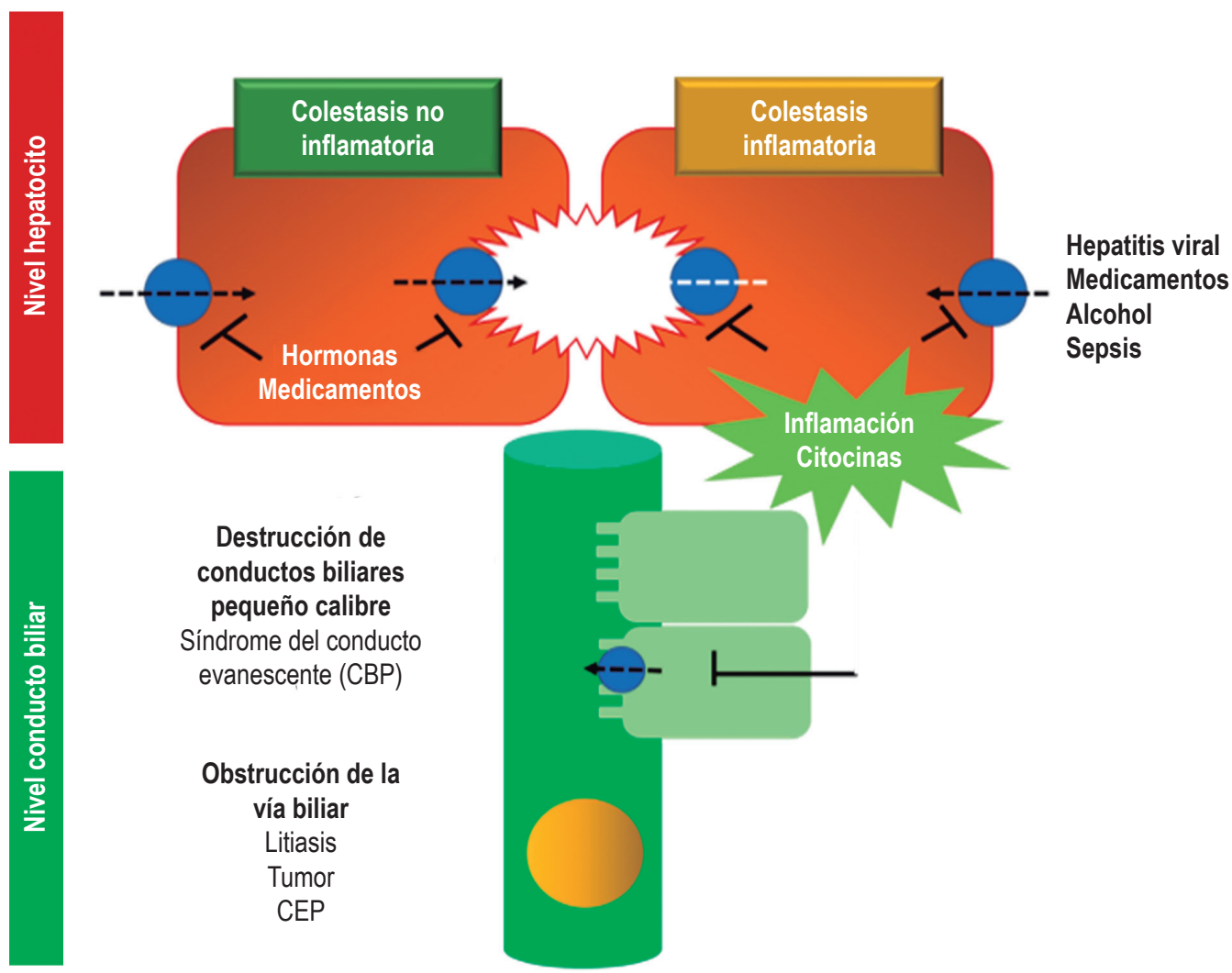

Figura 2. Mecanismos principales de la colestasis. CBP: colangitis biliar primaria; CEP: colangitis esclerosante primaria. Tomada de la referencia 5 .

los 3 primeros meses de vida y la pubertad, y se incrementa de forma gradual entre los 40 y 65 años, especialmente en mujeres y durante el embarazo $(2,10)$. Mientras tanto, los adolescentes hombres pueden alcanzar entre 2 y 5 veces el valor normal de los adultos, lo que se correlaciona con el crecimiento óseo (15).

Otras variaciones fisiológicas pueden encontrarse en los afroamericanos, que tienen entre un 10 y un $15 \%$ más de niveles séricos de ALP, así como en los fumadores, que pueden alcanzar hasta un $10 \%$ más, en comparación con las personas no fumadoras (10). De igual forma, las personas con un grupo sanguíneo $\mathrm{O}$ y $\mathrm{B}$ pueden presentar una elevación de la ALP, luego de una comida con alto contenido de grasa, a raíz del influjo intestinal de la misma enzima $(2,10,11)$.

Las causas patológicas que generan un incremento de la ALP varían desde enfermedades óseas como fracturas, enfermedad de Paget, osteomalacia, deficiencia de vitamina D, falla cardíaca o renal, hipertiroidismo e hiperparatiroidismo, hasta neoplasias hematológicas como leucemia y linfoma, o de células renales (10).

Este fenómeno surge tanto por la presencia de isoformas específicas en el tejido, como las placentarias (placental- like alkaline phosphatase, PLALP, o la isoenzima Regan), las intestinales (Intestinal Alkaline Phosphatase, IALP), las de hígado/hueso/renal (Liver/Bone/Kidney, ALP) y las células germinales de ALP (Germ Cell Alkaline Phosphatase, GCALP, o la isoenzima NAGAO), así como por su localización en la superficie celular, lo cual hidroliza los ésteres de monofosfato a $\mathrm{pH}$ alto, con la liberación de fosfato inorgánico (16-18).

Ante la presencia de una ALP elevada, se debe considerar si este incremento tiene una fuente intrahepática o extrahepática (principalmente en los huesos, el intestino, los leucocitos y la placenta). Por tanto, se requiere la toma de gamma-glutamil transferasa o una medición de las isoenzimas de ALP que considere un origen óseo $(2,11)$. Esta actividad ósea cobija alrededor de la mitad de la ALP en los adultos, por lo que esta es la isoenzima más importante y útil para su estudio (18).

No obstante, la gamma-glutamil transferasa presenta una baja especificidad para enfermedades colestásicas, dado que puede elevarse hasta en un $50 \%$ de los pacientes alcohólicos sin evidencia de enfermedad hepática (19). Esto también puede ocurrir en otras etiologías entre las que se 
incluyen enfermedades pancreáticas, infarto de miocardio (20), falla renal y enfisema (21), y en pacientes que consumen medicamentos como la fenitoína y los barbitúricos.

En consecuencia, este no es un examen que deba ser usado para la tamización de enfermedades hepáticas subyacentes, en ausencia de anormalidad en las otras pruebas hepáticas (15). La bilirrubina sérica, en su forma conjugada, puede estar elevada (o no) en presencia de una colestasis $(2,22,23)$.

\section{ETIOLOGÍA}

Dentro de las múltiples causas de colestasis pueden identificarse alteraciones genéticas que aparecen en la infancia y trastornos autoinmunes, sistémicos o secundarios a medicamentos. Sin embargo, una forma de categorizar la etiología es dividirla en causas intrahepáticas y extrahepáticas (3).

Las causas intrahepáticas son ocasionadas, generalmente, por condiciones inflamatorias y destructivas que suelen denominarse como el síndrome del conducto evanescente $(3,5)$. La colangitis biliar primaria (CBP), anteriormente conocida como cirrosis biliar primaria, es una de las causas más frecuentes de colestasis de origen autoinmune y genera una colangitis granulomatosa linfocítica que involucra a los conductos biliares de pequeño calibre $(3,24,25)$.

Por lo general, la CBP se presenta en mujeres (relación 9:1) entre los 40 y 60 años; se acompaña de anticuerpos antimitocondriales (Anti-mitochondrial antibodies, AMA) positivos (títulos $>1: 40$ ) dirigidos contra la subunidad E2 de la piruvato deshidrogenasa (22). Su diagnóstico se realiza a partir de 2 de 3 criterios: la presencia de colestasis por elevación de la ALP, los AMA positivos y la evidencia histopatológica de colangitis no supurativa con destrucción de los conductos biliares interlobulares de mediano y pequeño calibre (26).

Entre tanto, la colangitis esclerosante primaria (CEP) es una entidad que predomina en los hombres 2:1 con respecto a las mujeres y tiene una edad media de aparición de, aproximadamente, 40 años. Esta afecta los conductos biliares intrahepáticos y extrahepáticos, lo que genera estrecheces, con posterior fibrosis y cirrosis $(27,28)$. El diagnóstico de esta enfermedad involucra una combinación de criterios por imágenes que pueden mostrar estenosis localizadas a lo largo de todo el árbol biliar, las cuales son identificables mediante una colangioresonancia o una colangiopancreatografía retrógrada endoscópica. Los cambios en el conducto biliar pueden observarse a partir de la biopsia hepática, en la que se evidencia una fibrosis concéntrica periductal o en «piel de cebolla» (27).

Asimismo, se pueden identificar anticuerpos antimúsculo liso (anti-smooth muscle antibody, ASMA), anticuerpos antinucleares (AntiNuclear Antibodies, ANA) y anti- cuerpos contra el citoplasma de neutrófilos (Antineutrophil Cytoplasmic Antibodies, ANCA) hasta en un $50 \%$ de los pacientes; sin embargo, estos no son específicos de dicha entidad (28).

Otras enfermedades con sustrato autoinmune, que pueden generar colestasis y que comparten la misma fisiopatología de la fibrosis periductal y la pérdida progresiva de los conductos biliares, son la colangiopatía por inmunoglobulina G4; el rechazo crónico ductopénico en trasplante hepático y la enfermedad de injerto contra huésped, la cual aparece en los primeros 100 días luego del trasplante alogénico de células hematopoyéticas.

Incluso el linfoma (tanto Hodgkin como no Hodgkin) podría manifestarse hasta en un $10 \%$ de los pacientes con ictericia y ductopenia progresiva (síndrome de conducto evanescente) y en un $40 \%$ de aquellos con niveles elevados de ALP. Se acompaña de los síntomas clásicos B como fiebre y pérdida de peso, y presenta un espectro que abarca desde una lesión solitaria hasta el compromiso infiltrativo difuso, lo que responde tanto a la quimioterapia como a la radioterapia $(3,29)$.

El uso de medicamentos puede generar lesiones hepáticas (enfermedad hepática inducida por fármacos [Drug Induced Liver Injury, DILI] ) y desembocar en una falla hepática fulminante ( $13 \%$ de los pacientes en Estados Unidos). Estas lesiones se manifiestan con un patrón hepatocelular, y una elevación de transaminasas, o un patrón colestásico con elevación de la ALP $>2$ veces el límite superior de la normalidad (30).

Así, los cambios generados por los medicamentos pueden alcanzar desde una discreta inflamación del parénquima y ductopenia leve hasta una inflamación progresiva, fibrosis y la pérdida de los conductos biliares (31). Entre los principales fármacos identificados como desencadenantes de este fenómeno, se encuentran los antibióticos (amoxicilina/clavulanato, nitrofurantoína, isoniazida y ciprofloxacina, entre otros), agentes del sistema nervioso central (SNC) (valproato, fenitoína, metildopa, lamotrigina) y medicamentos usados en los trastornos endocrinos (propiltiouracilo, atorvastatina, troglitazona) y cardiovasculares (amiodarona) (32).

Por otra parte, el embarazo facilita la presencia de colestasis y genera dos patologías acompañadas de alteraciones en el perfil hepático. Una de ellas es la hiperémesis gravídica, que ocurre durante el primer trimestre del embarazo, y otra es la colestasis intrahepática del embarazo, que aparece en el segundo y el tercer trimestre de la gestación (33). Esta última se manifiesta con prurito, una elevación de los ácidos biliares séricos (niveles $>40 \mathrm{mmol} / \mathrm{L}$ se asocian con una mayor tasa de complicaciones fetales) y la resolución espontánea de los síntomas entre las semanas 4 y 6 posteriores al parto $(30,34)$. 
De igual forma, existe una particularidad en las mujeres que consumen anticonceptivos orales, dado que este síndrome se presenta en mujeres heterocigotas para la deficiencia de MDR3, el cual es un translocador en el canalículo de la membrana del hepatocito (35).

La nutrición parenteral total también se ha descrito como una causa de colestasis (26). En efecto, en los pacientes que reciben este tratamiento se han identificado modificaciones hemodinámicas dentro del acino y los colangiolos, desde la arteria hepática, en lugar de ser recibidos por la vena porta. Asimismo, los efectos del ayuno prolongado o el reposo digestivo generan modificaciones en la circulación enterohepática y cambios en la composición nutricional aportada, que no será exactamente igual a la nutrición enteral $(36,37)$.

La sepsis también se ha descrito como una causa de colestasis que involucra a los pacientes con infecciones bacterianas, tanto por gérmenes Gram positivos como Gram negativos, mediante la liberación de endotoxinas bacterianas, como los lipopolisacáridos, que reducen la recaptura de la bilis en los receptores de sodio-taurocolato y en los transportadores que liberan las sales biliares.

Dicho mecanismo está mediado por la presencia de citocinas proinflamatorias como el interferón gamma y el factor de necrosis tumoral, lo cual facilita la infiltración linfocítica en el epitelio ductal y reduce la secreción de bilis en los conductos biliares $(38,39)$. Este fenómeno puede manifestarse con hiperbilirrubinemia y alcanzar niveles $>20 \mathrm{mg} / \mathrm{L}$ dentro de las primeras $48 \mathrm{~h}$, así como también puede incrementarse por cargas aumentadas de bilirrubina (hemólisis trauma o hematoma) y por alteraciones hepáticas en el flujo biliar (coledocolitiasis o inflamación intraductal) (40).

Los trastornos sistémicos como la sarcoidosis, que inicialmente genera un compromiso granulomatoso pulmonar, y los nódulos pulmonares, que aparecen en personas entre los 20 y 40 años, se acompañan de dolor abdominal, náuseas, vómito, una elevación de la ALP y de la enzima convertidora de angiotensina (39).

Otras causas del compromiso sistémico con colestasis son las infecciones por virus, como el Epstein-Barr y el citomegalovirus. Por la naturaleza de su $\mathrm{ADN}$, estos virus comprometen el hígado y, en el caso del citomegalovirus, su presencia es capaz de generar una colangiopatía por VIH en pacientes con infección por retrovirus. Esta constituye una causa inusual de colangitis esclerosante $(3,39)$.

De forma simultánea, las enfermedades de depósito de inmunoglobulinas, como la amiloidosis primaria (por neoplasias de células plasmáticas como la macroglobulinemia o el mieloma múltiple) y secundaria (por enfermedades inflamatorias sistémicas como la artritis reumatoide, la tuberculosis y la osteomielitis) también se han considerado como causantes de colestasis -la cual es inicialmente identificada por un aumento de la ALP-y de hepatomegalia $(29,39)$.

Los trastornos genéticos que involucran defectos en el transporte hepatocelular de la bilis han sido descritos como causantes de colestasis. Entre estos trastornos se incluye la colestasis intrahepática progresiva familiar (Progressive Familial Intrahepatic Cholestasis, PFIC), la cual hace parte de los trastornos autosómicos recesivos de aparición desde el período neonatal. Dicha alteración se presenta con colestasis de aparición en el primer mes de vida, junto con una ALP elevada y una gamma-glutamil transferasa normal, tanto en la PFIC 1 como en la PFIC 2.

Esta última causa una falla hepática con progresión a cirrosis hepatocarcinoma y colangiocarcinoma, mientras que la PFIC 3 tiene una presentación más tardía en la infancia, acompañada de una gamma-glutamil elevada $(30,41)$.

Entre tanto, la colestasis intrahepática benigna recurrente se caracteriza por episodios repetidos de prurito e ictericia, que duran semanas o meses, y están acompañados de una ALP elevada, posterior al inicio de estos episodios de prurito con un pronóstico benigno (42). Por su parte, el síndrome de Alagille es un trastorno autosómico dominante, con compromiso multisistémico (hígado, corazón, sistema óseo y ojos), que involucra una mutación en el gen JAG1 y se manifiesta con ictericia, hiperbilirrubinemia y una ALP elevada.

Otra entidad menos frecuente es la fibrosis quística secundaria a la mutación del gen que involucra a los canales transmembrana conductores de cloro (CFTR), los cuales modifican el trasporte aniónico, así como la depuración del moco, y presentan un compromiso respiratorio con bronquiectasias y en el hígado, con cirrosis (43).

Además de la fibrosis quística, el síndrome de DubinJohnson se manifiesta mediante la ictericia y la hiperbilirrubinemia predominantemente directa, $y$ con cambios en la coloración del hígado, la cual se torna de un aspecto negro, por causa de una deficiente expresión del transportador MRP2 que altera la excreción de la bilis $(10,44)$.

\section{DIAGNÓSTICO}

El abordaje de la colestasis parte de la historia clínica y del examen físico, con los cuales se indican los antecedentes médicos y se establece si existen precedentes familiares de colestasis (lo que sugiere una colestasis intrahepática familiar) (42), antecedentes quirúrgicos de colecistectomía (probable coledocolitiasis) e incluso se indaga sobre las terapias farmacológicas usadas y el consumo de remedios herbales y de alcohol, que podrían ser causas de esta afección $(3,9,10)$.

Durante el examen físico, la presencia de ictericia indolora, con o sin masa palpable en el hipocondrio derecho, puede sugerir una obstrucción maligna, mientras que la coledocolitiasis puede presentarse con ictericia y dolor 
abdominal. También se puede manifestar con colangitis (fiebre, ictericia, dolor en el hipocondrio derecho), por causa de una obstrucción parcial o total de la vía biliar. Entre tanto, la colestasis intrahepática puede aparecer junto con prurito y fatiga.

Así, el primer paso para estudiar la colestasis es la medición de la ALP, la cual no es específica del hígado, sino que puede encontrarse en los huesos, el riñón, la placenta y en los glóbulos blancos. En caso de hallarse una elevación de la ALP sin un aumento de transaminasas o hiperbilirrubinemia, y sin evidencias imagenológicas que sugieran la existencia de un componente obstructivo, sería útil la medición de la isoenzima ósea por electroforesis o 5'-nucleotidasa y gamma-glutamil transferasa. También puede resultar oportuno considerar las elevaciones mismas de la ALP en el transcurso de la vida y la variación según el género, dado que esta es más elevada en las mujeres $(33,34)$.

Del mismo modo, se pueden complementar los estudios a partir de la toma de serologías virales, a fin de excluir el diagnóstico de hepatitis de etiología infecciosa. Las etiologías autoinmunes deben descartarse por medio de la medición de los ANA, los ASMA, los AMA, los ANCA de fracción perinuclear y los niveles de inmunoglobulina G y M (45).

De forma simultánea, las imágenes diagnósticas permiten orientar la colestasis de origen intrahepático o extrahepático. La ultrasonografía abdominal es fundamental para excluir la presencia de una obstrucción biliar extrahepática (10). Sin embargo, su uso tiene dificultades técnicas en personas obesas, dado que omite hasta el $60 \%$ de los cálculos en el colédoco de este tipo de pacientes (46).

Otros estudios complementarios en la vía biliar son la tomografía de abdomen -aunque esta no delinea de forma adecuada la vía biliar- (47), la colangioresonancia magnética, la ultrasonografía endoscópica o ecoendoscopia y la colangiopancreatografía retrógrada endoscópica. Estas herramientas son fundamentales para la identificación de las lesiones causantes de la obstrucción extrahepática.

Las dos primeras pruebas permiten una caracterización de la vía biliar intrahepática, mientras que la colangiopancreatografía retrógrada endoscópica contribuye a la instrumentación de la vía biliar, a la extracción de los cálculos en ella y a la toma de biopsias ante la sospecha de lesiones neoplásicas de los conductos. Asimismo, ayudan a la dilatación de la estenosis de la vía biliar y a la instalación de endoprótesis para permeabilizar el colédoco, en casos de estrechez clínicamente manifiesta (10) (Figura 3).

\section{TRATAMIENTO}

La CBP y la CEP son los prototipos de las enfermedades crónicas colestásicas y, por tanto, se consideran los trastornos modelo para discutir el manejo médico de la colestasis (48). Las estrategias de tratamiento buscan limitar la acumulación de ácidos biliares y reducir la reserva de estos, y apuntan a la hepatoprotección a partir de inducir coleresis o la excreción biliar de dichos ácidos, lo cual limita el daño del colangiocito y modula la inflamación generada por los ácidos biliares (49).

Así pues, el manejo de las enfermedades colestásicas (principalmente la CBP y la CEP) se sustenta en el uso de ácido ursodesoxicólico, el cual estimula el flujo biliar y de bicarbonato en los hepatocitos y en los colangiocitos. Además, brinda un efecto antiapoptótico y antiinflamatorio (50) para identificar los criterios de respuesta, luego del inicio de dicho tratamiento en la CBP (51) (Tabla 1).

Sin embargo, en la CEP no existe suficiente evidencia que demuestre un beneficio para la supervivencia de los pacientes, pese a una mejoría en el perfil hepático (23). Otros agentes que aún se encuentran en uso son los agonistas de los receptores farnesoide X (Farnesoid X Receptor,

Tabla 1. Criterios de respuesta luego del tratamiento de la CBP.

\begin{tabular}{llcc}
\hline & \multicolumn{1}{c}{ Definición } & $\begin{array}{c}\text { Tiempo de } \\
\text { seguimiento }\end{array}$ & \multicolumn{1}{c}{$\begin{array}{c}\text { Desenlace clínico } \\
\text { respuesta }\end{array}$} \\
\hline $\begin{array}{llcl}\text { Barcelona } \\
\text { Paris } 1\end{array}$ & Descenso $>40 \%$ de la ALP o normalización. & 12 meses & Supervivencia libre de trasplante. \\
& $\begin{array}{l}\text { Descenso de ALP } \leq 3, \text { límite superior normalidad y AST } \\
\leq 2, \text { límite superior en normalidad y bilirrubina normal. }\end{array}$ & 12 meses & Supervivencia libre de trasplante. \\
Paris 2 & Descenso de ALP y AST $\leq 1,5$, límite superior en & 12 meses & Supervivencia libre de trasplante y de \\
& normalidad y bilirubina normal. & & complicaciones, progresión a cirrosis. \\
Róterdam & Bilirubina normal o albúmina. & 12 meses & Supervivencia libre de trasplante. \\
Toronto & ALP $\leq 1,67$, límite superior en normalidad. & 24 meses & Progresión histológica. \\
\hline
\end{tabular}

ALP: fosfatasa alcalina (Alkaline Phosphatase); AST: aspartato aminotransferasa (Aspartate Aminotransferase). Modificada de la referencia 51. 


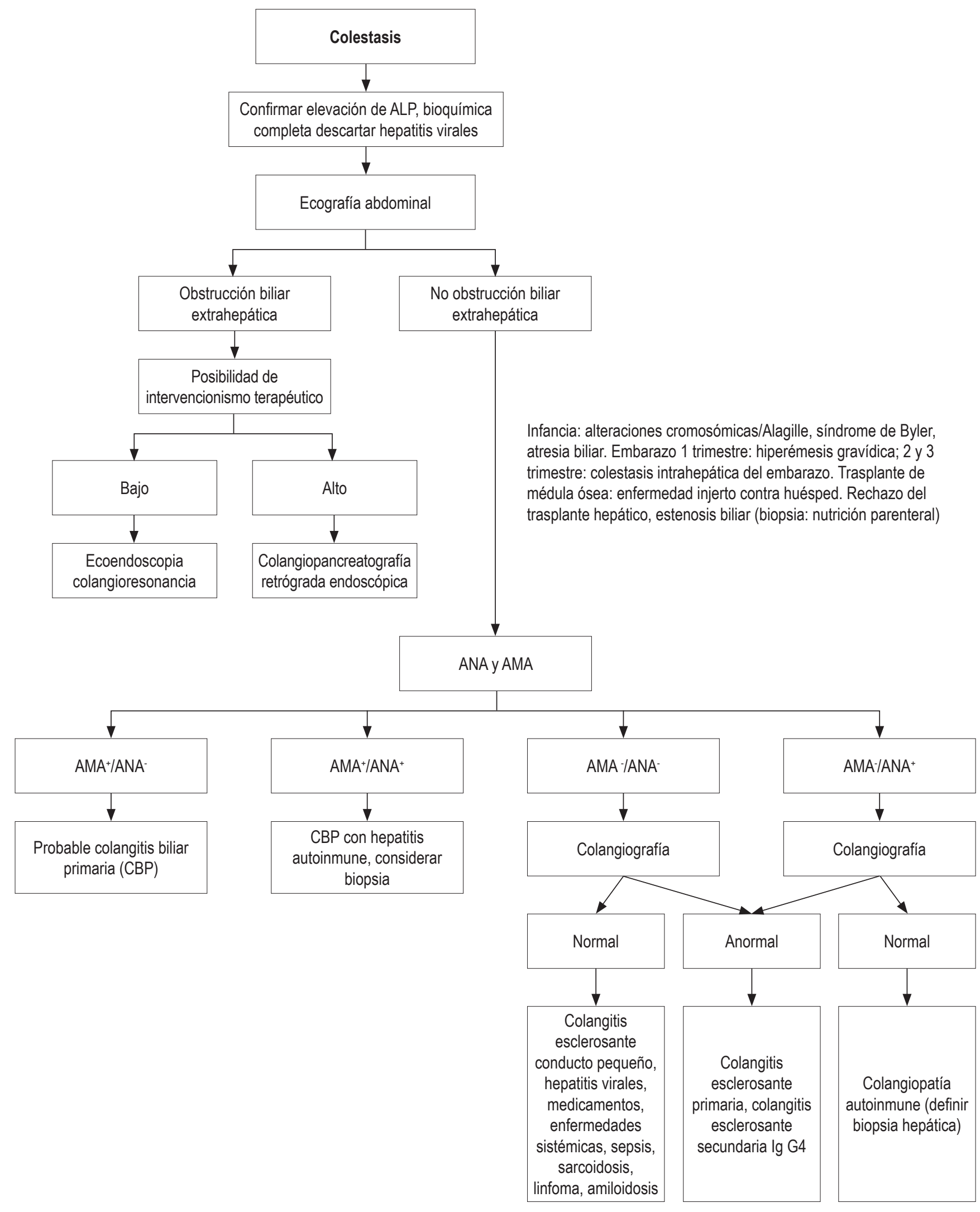

Figura 3. Algoritmo diagnóstico de colestasis. ALP: fosfatasa alcalina (Alkaline Phosphatase); ANA: anticuerpos antinucleares (AntiNuclear Antibodies); AMA: anticuerpos antimitocondriales (Anti-mitochondrial antibodies); Ig G4: inmunoglobulina G4. Modificada de la referencia 3. 
FXR) -como el ácido obeticólico-, el receptor retinoide $\mathrm{X}$ (Receptor X Retinoide, RXR), el receptor pregnano X (Pregnane X Receptor, PXR), el receptor de glucocorticoide (GR) y el receptor a del proliferador activado de peroxisomas (Perosyxomel Proliferator Activated Receptors, PPAR a).

En estos receptores actúan los fibratos como el fenofibrato y el bezafibrato, los cuales son modificadores transcripcionales de la formación de bilis (52) y previenen la acumulación de ácidos biliares, lo que reduce la importación y el aumento de la exportación de estas moléculas en los hepatocitos (49).

En caso de que se presenten pacientes sin respuesta al uso de medidas farmacológicas, se debe considerar el trasplante hepático, el cual alcanza supervivencias a 1 y 5 años del 83 y el $78 \%$, respectivamente. No obstante, existe la posibilidad de que haya recurrencia de la enfermedad, la cual puede alcanzar hasta un $8 \%$ luego de 5 años del trasplante, y un $22 \%$ a los 10 años de haber realizado el procedimiento (24).

\section{CONCLUSIONES}

La colestasis es un síndrome que compromete la síntesis, la secreción y el flujo de la bilis y puede derivar en riesgo de cirrosis e hipertensión portal. Es importante su diagnóstico temprano y la identificación de manifestaciones sutiles como el prurito y los cambios en los niveles de ALP, para lo cual es necesario tener claridad del origen hepático y excluir otras fuentes, dada su presencia en otros tejidos.

Así pues, el tratamiento busca disminuir la inflamación generada por los ácidos biliares y modificar la reserva de estos, bien sea por su excreción o por el bloqueo de la circulación enterohepática. En consecuencia, es fundamental excluir las complicaciones causadas por esta alteración como la presencia de osteoporosis y la deficiencia de vitaminas, y tratarlas oportunamente.

\section{Conflicto de intereses}

Los autores no declaran ningún conflicto de interés.

\section{Fuente de financiación}

Ninguna.

\section{Agradecimientos}

Ninguno declarado por los autores.

\section{REFERENCIAS}

1. McIntyre N. Cholestasis. En: Bircher J, Benhamou JP, McIntyre N, Rizzeto M, Rodés J. Oxford Textbook of
Clinical Hepatology. Oxford: Oxford Medical Publications, $2^{\text {a }}$ edición; 1999. p. 1574-9.

2. Heathcote EJ. Diagnosis and management of cholestatic liver disease. Clin Gastroenterol Hepatol. 2007;5(7):77682. https://doi.org/10.1016/j.cgh.2007.05.008

3. Pérez Fernández T, López Serrano P, Tomás E, Gutiérrez ML, Lledó JL, Cacho G, et al. Diagnostic and therapeutic approach to cholestatic liver disease. Rev Esp Enferm Dig. 2004;96(1):60-73. https://doi.org/10.4321/S113001082004000100008

4. Trauner M, Meier PJ, Boyer JL. Molecular pathogenesis of cholestasis. N Engl J Med. 1998;339(17):1217-27. https:// doi.org/10.1056/NEJM199810223391707

5. Zollner G, Trauner M. Mechanisms of cholestasis. Clin Liver Dis. 2008;12(1):1-26, vii. https://doi.org/10.1016/j. cld.2007.11.010

6. Elferink RO. Cholestasis. Gut. 2003;52 Suppl 2(Suppl 2):ii42-ii48. https://doi.org/10.1136/gut.52.suppl_2.ii42

7. Meier PJ. Molecular mechanisms of hepatic bile salt transport from sinusoidal blood into bile. Am J Physiol. 1995;269(6 Pt 1):G801-12. https://doi.org/10.1152/ ajpgi.1995.269.6.G801

8. Hofmann AF, Hagey LR. Bile acids: chemistry, pathochemistry, biology, pathobiology, and therapeutics. Cell Mol Life Sci. 2008;65(16):2461-83. https://doi.org/10.1007/ s00018-008-7568-6

9. Maillette de Buy Wenniger L, Beuers U. Bile salts and cholestasis. Dig Liver Dis. 2010;42(6):409-18. https://doi. org/10.1016/j.dld.2010.03.015

10. Siddique A, Kowdley KV. Approach to a patient with elevated serum alkaline phosphatase. Clin Liver Dis. 2012;16(2):199_ 229. https://doi.org/10.1016/j.cld.2012.03.012

11. Assis DN. Chronic Complications of Cholestasis: Evaluation and Management. Clin Liver Dis. 2018;22(3):533-544. https://doi.org/10.1016/j.cld.2018.03.014

12. Shen YM, Wu JF, Hsu HY, Ni YH, Chang MH, Liu YW, et al. Oral absorbable fat-soluble vitamin formulation in pediatric patients with cholestasis. J Pediatr Gastroenterol Nutr. 2012;55(5):587-91. https://doi.org/10.1097/ MPG.0b013e31825c9732

13. Shneider BL, Magee JC, Bezerra JA, Harber B, Karpen SJ, Raghunathan T, et al.Efficacy of fat-soluble vitamin supplementation in infants with biliary atresia. Pediatrics. 2012;130(3):e607e614. https://doi.org/10.1097/MPG.0b013e31825c9732

14. Craddock AL, Love MW, Daniel RW, et al. Expression and transport properties of the human ileal and renal sodiumdependent bile acid transporter. Am J Physiol 1998; 274 (1): 157-69. https://doi.org/10.1152/ajpgi.1998.274.1.G157

15. Kwo PY, Cohen SM, Lim JK. ACG Clinical Guideline: Evaluation ofAbnormalLiverChemistries.AmJGastroenterol. 2017;112(1):18-35. https://doi.org/10.1038/ajg.2016.517

16. Schiele F, Henny J, Hitz J, Petitclerc C, Gueguen R, Siest G. Totalbone and liver alkaline phosphatases in plasma: biological variations and reference limits. Clin Chem. 1983;29(4):63441. https://doi.org/10.1093/clinchem/29.4.634 
17. Stigbrand T. Present status and future trends of human alkaline phosphatases. Prog Clin Biol Res. 1984;166:3-14.

18. Sharma U, Pal D, Prasad R. Alkaline phosphatase: an overview. Indian J Clin Biochem. 2014;29(3):269-278. https:// doi.org/10.1007/s12291-013-0408-y

19. Moussavian SN, Becker RC, Piepmeyer JL, Mezey E, Bozian RC. Serum gamma-glutamyl transpeptidase and chronic alcoholism. Influence of alcohol ingestion and liver disease. Dig Dis Sci. 1985;30(3):211-4. https://doi.org/10.1007/ BF01347885

20. Lee DH, Silventoinen K, Hu G, Jacobs DR, Jousilahti P, Sundvall J, et al. Serum gamma-glutamyltransferase predicts non-fatal myocardial infarction and fatal coronary heart disease among 28,838 middle-aged men and women. Eur Heart J. 2006;27(18):2170-6. https://doi.org/10.1093/ eurheartj/ehlo86

21. Kim HW, Lee SH, Lee DH. Relationship of serum gammaglutamyltransferase levels with pulmonary function and chronic obstructive pulmonary disease. Lung. 2014;192(5):71927. https://doi.org/10.1007/s00408-014-9616-3

22. European Association for the Study of the Liver. EASL Clinical Practice Guidelines: The diagnosis and management of patients with primary biliary cholangitis. J Hepatol. 2017;67(1):145-172. https://doi.org/10.1016/j. jhep.2017.03.022

23. European Association for the Study of the Liver. EASL Clinical Practice Guidelines: management of cholestatic liver diseases. J Hepatol. 2009;51(2):237-67. https://doi. org/10.1016/j.jhep.2009.04.009

24. Patel A, Seetharam A. Primary Biliary Cholangitis: Disease Pathogenesis and Implications for Established and Novel Therapeutics. J Clin Exp Hepatol. 2016;6(4):311-318. https://doi.org/10.1016/j.jceh.2016.10.001

25. Lindor KD, Gershwin ME, Poupon R, Kaplan M, Bergasa NV, Heathcote EJ, et al. Primary biliary cirrhosis. Hepatology. 2009;50(1):291-308. https://doi.org/10.1002/hep.22906

26. Jansen PL, Ghallab A, Vartak N, Reif R, Schaap FG, Hampe $\mathrm{J}$, et al. The ascending pathophysiology of cholestatic liver disease. Hepatology. 2017;65(2):722-738. https://doi. org/10.1002/hep.28965

27. Williamson KD, Chapman RW. New Therapeutic Strategies for Primary Sclerosing Cholangitis. Semin Liver Dis. 2016;36(1):5-14. https://doi.org/10.1055/s-0035-1571274

28. Lindor KD, Kowdley KV, Harrison ME; American College of Gastroenterology. ACG Clinical Guideline: Primary Sclerosing Cholangitis.Am JGastroenterol.2015;110(5):64659. https://doi.org/10.1038/ajg.2015.112

29. Smit WL, Culver EL, Chapman RW. New Thoughts on Immunoglobulin G4-Related Sclerosing Cholangitis. Clin Liver Dis. 2016;20(1):47-65. https://doi.org/10.1016/j. cld.2015.08.004

30. Nguyen KD, Sundaram V, Ayoub WS. Atypical causes of cholestasis. World J Gastroenterol. 2014;20(28):9418-26. http://dx.doi.org/10.3748/wjg.v20.i28.9418
31. Bhamidimarri KR, Schiff E. Drug-induced cholestasis. Clin Liver Dis. 2013;17(4):519-31, vii. https://doi. org/10.1016/j.cld.2013.07.015

32. Padda MS, Sanchez M, Akhtar AJ, Boyer JL. Drug-induced cholestasis. Hepatology. 2011;53(4):1377-1387. https:// doi.org/10.1002/hep.24229

33. Floreani A, Gervasi MT. New Insights on Intrahepatic Cholestasis of Pregnancy. Clin Liver Dis. 2016;20(1):17789. https://doi.org/10.1016/j.cld.2015.08.010

34. Lammert F, Marschall HU, Glantz A, Matern S. Intrahepatic cholestasis of pregnancy: molecular pathogenesis, diagnosis and management. J Hepatol. 2000;33(6):1012-21. https:// doi.org/10.1016/S0168-8278(00)80139-7

35. Jacquemin E, De Vree JM, Cresteil D, Sokal EM, Sturm E, Dumont $\mathrm{M}$, et al. The wide spectrum of multidrug resistance 3 deficiency: from neonatal cholestasis to cirrhosis of adulthood. Gastroenterology. 2001;120(6):1448-58. https://doi.org/10.1053/gast.2001.23984

36. Guglielmi FW, Regano N, Mazzuoli S, Fregnan S, Leogrande $\mathrm{G}$, Guglielmi A, et al. Cholestasis induced by total parenteral nutrition. Clin Liver Dis. 2008;12(1):97-110, viii. https:// doi.org/10.1016/j.cld.2007.11.004

37. Lauriti G, Zani A, Aufieri R, Cananzi M, Chiesa PL, Eaton $S$, et al. Incidence, prevention, and treatment of parenteral nutrition-associated cholestasis and intestinal failureassociated liver disease in infants and children: a systematic review. JPEN J Parenter Enteral Nutr. 2014;38(1):70-85. https://doi.org/10.1177/0148607113496280

38. Geier A, Fickert P, Trauner M. Mechanisms of disease: mechanisms and clinical implications of cholestasis in sepsis. Nat Clin Pract Gastroenterol Hepatol. 2006;3(10):57485. https://doi.org/10.1038/ncpgasthep0602

39. Delemos AS, Friedman LS. Systemic causes of cholestasis. Clin Liver Dis. 2013;17(2):301-17. https://doi. org/10.1016/j.cld.2012.11.001

40. Strnad P, Tacke F, Koch A, Trautwein C. Liver - guardian, modifier and target of sepsis. Nat Rev Gastroenterol Hepatol. 2017;14(1):55-66. https://doi.org/10.1038/ nrgastro.2016.168

41. Davit-Spraul A, Gonzáles E, Baussan C, Jacquemin E. The spectrum of liver diseases related to ABCB4 gene mutations: pathophysiology and clinical aspects. Semin Liver Dis. 2010;30(2):134-46. https://doi.org/10.1055/s-0030-1253223

42. Luketic VA, Shiffman ML. Benign recurrent intrahepatic cholestasis. Clin Liver Dis. 2004;8(1):133-49, vii. https:// doi.org/10.1016/S1089-3261(03)00133-8

43. Elborn JS. Cystic fibrosis. Lancet. 2016;388(10059):25192531. https://doi.org/10.1016/S0140-6736(16)00576-6

44. Memon N, Weinberger BI, Hegyi T, Aleksunes LM. Inherited disorders of bilirubin clearance. Pediatr Res. 2016;79(3):378-86. https://doi.org/10.1038/pr.2015.247

45. MarzoratiS, Invernizzi P,Lleo A. Making Sense of Autoantibodies in Cholestatic Liver Diseases. Clin Liver Dis. 2016;20(1):33-46. https://doi.org/10.1016/j.cld.2015.08.003 
46. Shea JA, Berlin JA, Escarce JJ, Clarke JR, Kinosian BP, Cabana MD, et al. Revised estimates of diagnostic test sensitivity and specificity in suspected biliary tract disease. Arch Intern Med. 1994;154(22):2573-81. https://doi. org/10.1001/archinte.1994.00420220069008

47. Balci NC, Befeler AS, Leiva P, Pilgram TK, Havlioglu N. Imaging of liver disease: comparison between quadruple-phase multidetector computed tomography and magnetic resonance imaging. J Gastroenterol Hepatol. 2008;23(10):1520-7. https://doi.org/10.1111/j.1440-1746.2008.05434.x

48. Samant H, Manatsathit W, Dies D, Shokouh-Amiri H, Zibari G, Boktor M, et al. Cholestatic liver diseases: An era of emerging therapies. World J Clin Cases. 2019;7(13):1571-1581. https://doi.org/10.12998/wjcc.v7.i13.1571
49. Arab JP, Cabrera D, Arrese M. Bile Acids in Cholestasis and its Treatment. Ann Hepatol.2017; 16(Suppl. 1: s3-105.):s53s57. https://doi.org/10.5604/01.3001.0010.5497

50. Beuers U, Trauner M, Jansen P, Poupon R. New paradigms in the treatment of hepatic cholestasis: from UDCA to FXR, PXR and beyond. J Hepatol. 2015;62(1 Suppl):S25-37. https://doi.org/10.1016/j.jhep.2015.02.023

51. Chazouillères O. Novel Aspects in the Management of Cholestatic Liver Diseases. Dig Dis. 2016;34(4):340-6. https://doi.org/10.1159/000444544

52. Czul F, Levy C. Novel Therapies on Primary Biliary Cirrhosis. Clin Liver Dis. 2016;20(1):113-30. https://doi. org/10.1016/j.cld.2015.08.006 